\title{
James Webb Space Telescope (JWST) Optical Telescope Element (OTE) Pathfinder Status and Plans
}

\author{
Lee D. Feinberg ${ }^{\mathrm{a}}$, Ritva Keski-Kuha ${ }^{\mathrm{a}}$, Charlie Atkinson ${ }^{\mathrm{b}}$, Andrew Booth ${ }^{\mathrm{c}}$, Tony Whitman ${ }^{\mathrm{d}}$ \\ ${ }^{a}$ NASA Goddard Space Flight Center, Greenbelt, MD, USA 20771; \\ ${ }^{\mathrm{b}}$ Northrop Grumman, Redondo Beach, CA 90278 USA \\ ${ }^{c}$ Sigma Space Corporation, Lanham, MD 20706 USA \\ ${ }^{\mathrm{d}}$ Exelis, Rochester, NY 14606 USA
}

\begin{abstract}
A JWST OTE Pathfinder telescope that includes two spare primary mirror segments, a spare secondary mirror, and a large composite structure with a deployed secondary support structure is in the assembly stage and will be fully completed this year. This Pathfinder will check out key steps in the ambient mirror integration process and also be used at the Johnson Space Center (JSC) to check out the optical Ground Support Equipment (GSE) and associated procedures that will be used to test the full JWST telescope and instruments at JSC. This paper will summarize the Pathfinder integration and testing flow, the critical Ground Support Equipment it will test and the key tests planned with the Pathfinder.
\end{abstract}

Keywords: Space Telescope, JWST, OTE, James Webb Space Telescope, Pathfinder

\section{INTRODUCTION}

NASA's James Webb Space Telescope (JWST) Optical Telescope Element (OTE) ${ }^{i}$ will be tested at the Johnson Space Center (JSC) after integration to the Integrated Science Instrument System (ISIM) as an integrated entity called the OTEISIM (OTIS). The testing at JSC will test both test the OTE internally and the OTIS interface including optical, thermal, electrical, software and operations tests and checkout. The test configuration is large and complex as it needs to test the primary mirror at center of curvature and measure the alignments of individual optical elements. Due to the size and complexity of the flight hardware and test configuration, a Pathfinder is being used to check out everything from integration processes to ground support equipment to test procedures.

In order to understand the plans for the Pathfinder, one needs to understand the basic test configuration. The flight OTIS test configuration is shown below in Figure 1. ${ }^{\text {ii iii }}$ All major test subsystems are through design and most key subsystems are either built or in manufacturing. For example, the Center of Curvature Object Assembly (COCOA) is built and has gone through cryo-optical testing at the Marshall Space Flight Center cryogenic chamber . The first Auto-Collimating Flat (ACF) is complete and will be installed for Pathfinder testing. The vibration isolation system including the six Minus $\mathrm{K}$ isolators, hanging configuration and photogrammetry will all be installed. All major optical GSE (OGSE) will be available for the first 2 pathfinder tests except for ACF's 2 and 3. ACF's 2 and 3 and the cryo thermal Ground Support Equipment (GSE) including the Space Vehicle Thermal System (SVTS) will be installed after the second pathfinder test (OGSE2) and prior to the Pathfinder thermal test.

Space Telescopes and Instrumentation 2014: Optical, Infrared, and Millimeter Wave, edited by Jacobus M. Oschmann, Jr., Mark Clampin, Giovanni G. Fazio, Howard A. MacEwen, Proc. of SPIE Vol. 9143, 91430E · @ 2014 SPIE

CCC code: $0277-786 \mathrm{X} / 14 / \$ 18 \cdot$ doi: $10.1117 / 12.2054782$ 


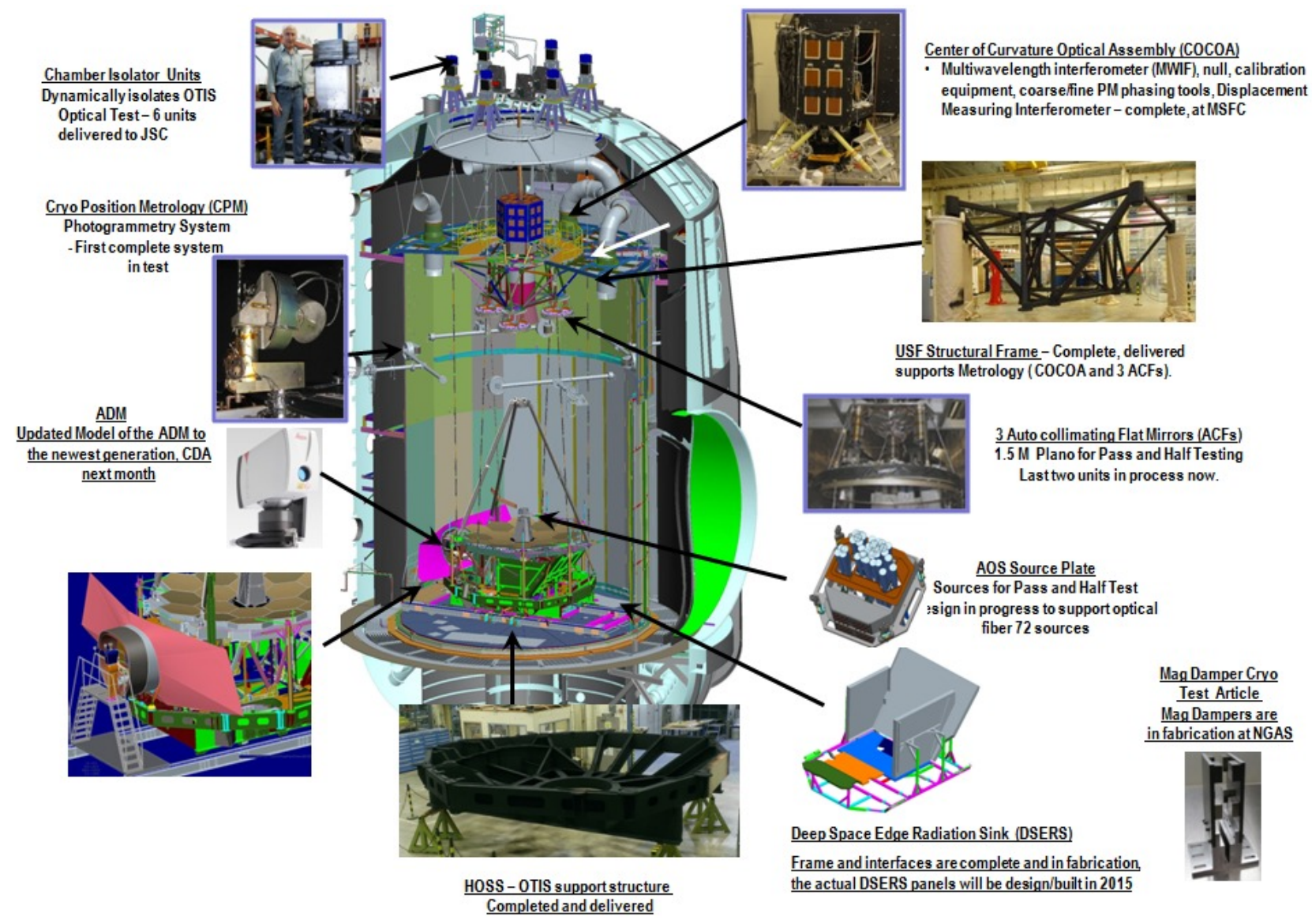

Figure 1: OTIS Test Configuration and Progress

The actual testing of the OTIS occurs in a single cryogenic test. However, prior to running the flight cryogenic test, extensive testing of the chamber and GSE is planned using the Pathfinder. As can be seen in the OTIS Integration and Testing Flow in Figure 2, GSE and test preparations continue through early 2014. This includes chamber commissioning tests down to Helium temperatures, cryo proofing, and a bakeout. Once complete, the chamber and GSE will be ready to accept the Pathfinder. The pathfinder testing shown below is then broken into three separate tests which incrementally test the optical GSE and then thermal GSE and procedures. After Pathfinder testing is complete, the flight OTIS testing will occur. Meanwhile, the flight OTIS flow occurs in parallel to chamber checkout. The key linkage is that the Aft Optics System of the Optical Telescope Element (OTE) is used during the OGSE2 test and then ships to Goddard for integration onto the OTE backplane. After the OTE is assembled it will be integrated to the Integrated Science Instrument Module (ISIM) to form OTIS. While still at Goddard, the OTIS undergoes ambient Center of Curvature testing on the primary mirror segments as part of pre and post vibration and acoustics checkout. Two new shaker tables are being procured for the OTIS test. All ambient integration, vibration, acoustics and CoC testing occurs in the same test complex at the Goddard Space Flight Center. After completing ambient environmental testing, the OTIS will be placed in a shipping container and flown to Houston on a C5 for OTIS testing in the JSC test chamber. 


\section{JWST OTIS Integration and Test}

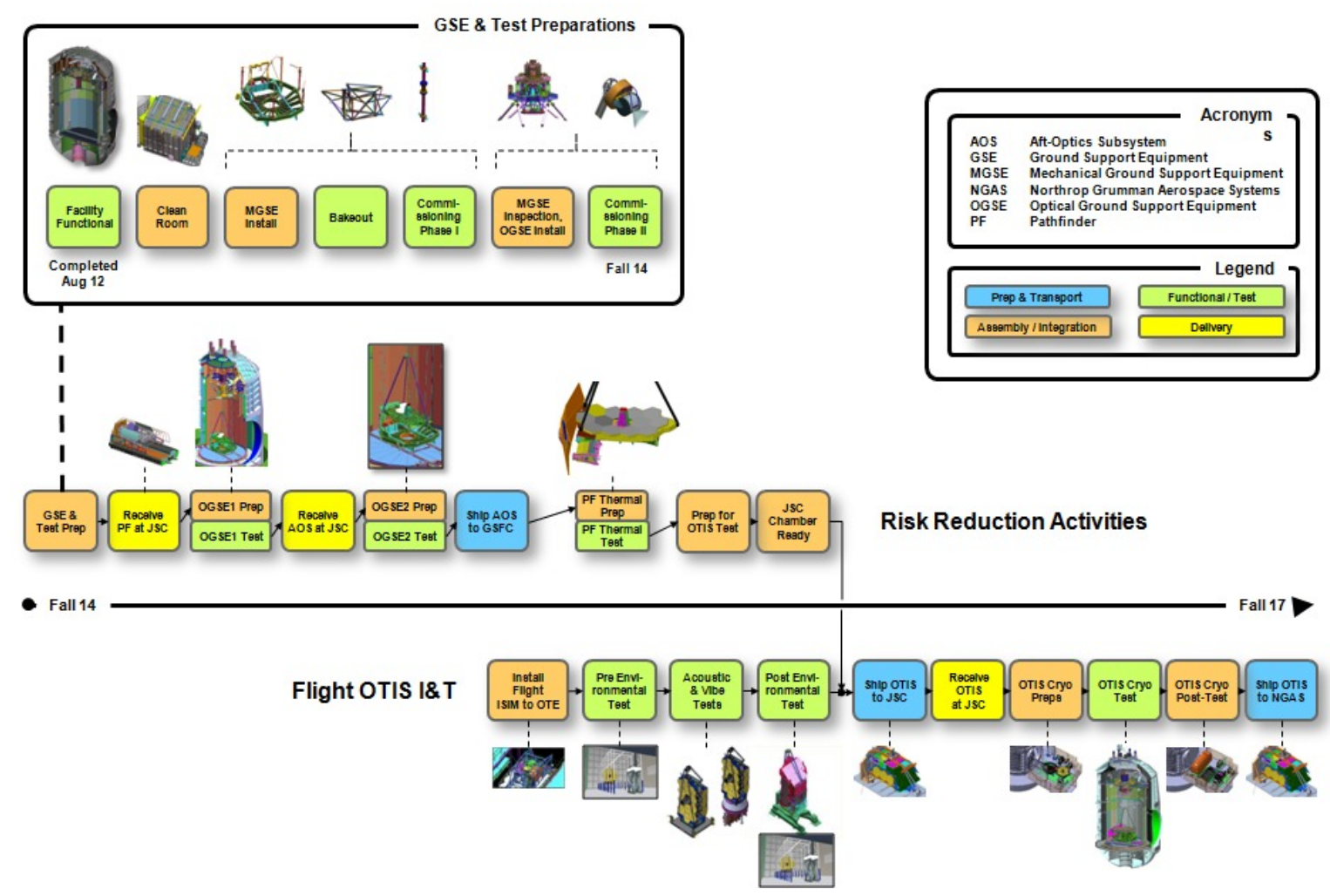

Figure 2: OTIS Integration and Test Flow

\section{PATHFinder}

With the test configuration and flow established, the Pathfinder itself can be understood. The Pathfinder configuration can be seen in Figure 3 and includes 2 spare Primary Mirror Segment Assemblies (PMSA's) and a spare Secondary Mirror Segment Assembly (SMSA). One PMSA, the engineering design unit (EDU), is a finished gold coated mirror. The other PMSA and the SMSA are partially polished and uncoated. However, the partially polished mirror figure is good enough for optical testing and well understood. The composite backplane was developed from drawings of the flight telescope and the Secondary Mirror Support Structure (SMSS) is also derived from flight designs. The backplane includes the composite backing structure that holds the mirrors. The Backplane Support Fixture (BSF) is aluminum and GSE that allows transport of the backplane but is removed prior to the cryogenic testing. As can be seen in the left portion of the figure, the SMSS is already built and installation of the mirror segments will start in summer of 2014. The Pathfinder will be sent to JSC, with the first cryogenic test in early 2015. 

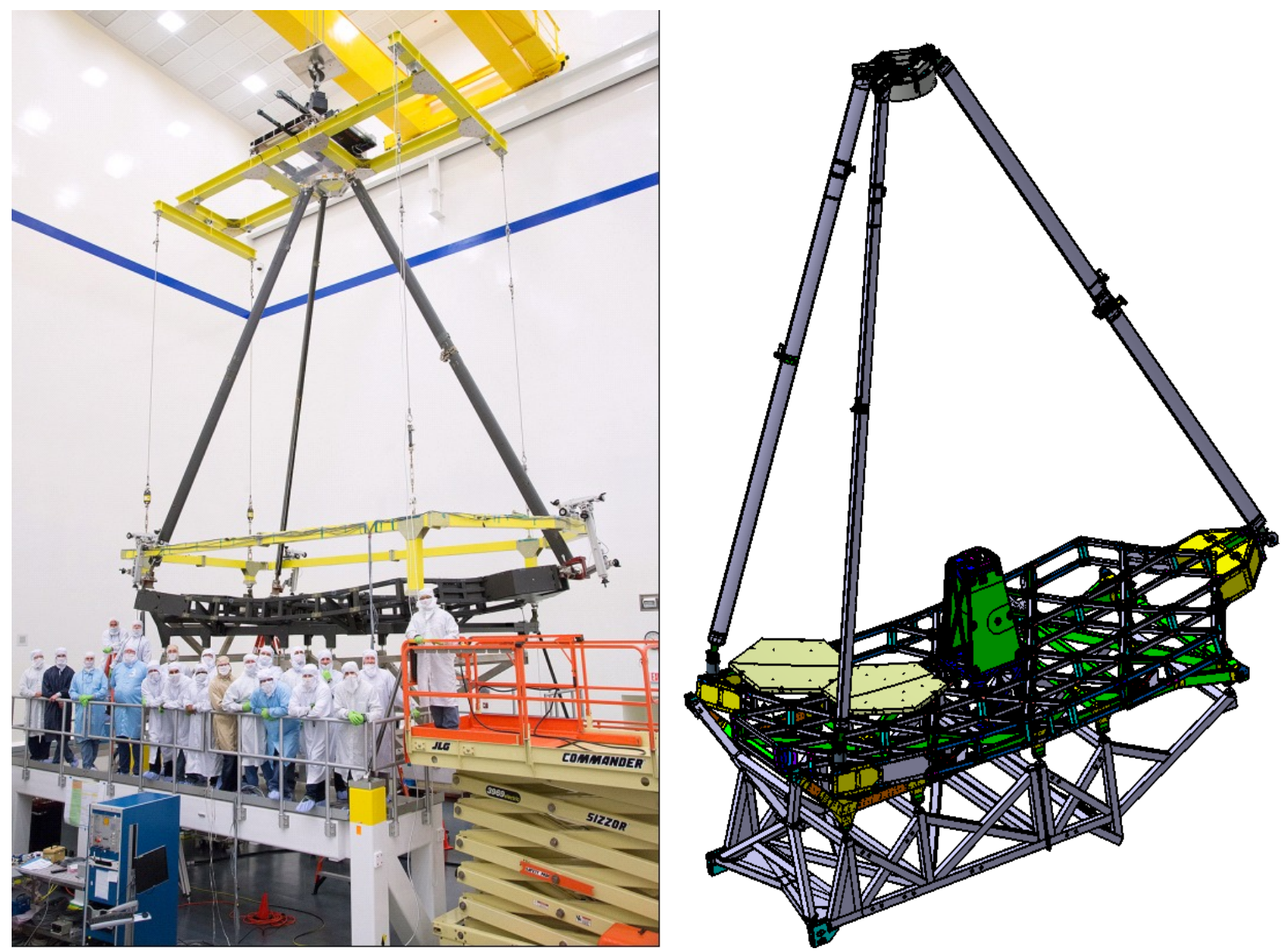

Figure 3: Pathfinder Structure and Configuration

Once OGSE testing begins, the Pathfinder will be used to check out all Optical GSE. ${ }^{\text {iv }}$ In addition to checking out optical GSE, the optical tests planned during flight OTIS testing will all be checked out to the extent possible. Table 1 shows a list of the optical tests planned and how these tests will be checked out during OGSE2 testing. In general, all tests that can be checked out during OGSE1 will be tested and then any tests not completed during OGSE1 but which can be tested during OGSE2 will be tested during OGSE2. The most important tests are cryogenic alignment tests as these are verification tests that can only occur during JSC testing. The checkout of cryo alignment measurement methods occurs during OGSE1 and OGSE2 incrementally. During OGSE1, the cryo photogrammetry ${ }^{v}$ and COCOA will be exercised and data taken to assure these tests will work during OTIS testing. During OGSE2, the AOS source plate assembly (ASPA), that provides the optical test sources for the OTIS cryo test will be exercised with the Beam Image Analyzer (BIA) to check out the OTE to ISIM alignment process. 


\begin{tabular}{|c|c|c|c|}
\hline Parameter & Test & Optical Test Equipment & Pathfinder Approach \\
\hline \multirow{3}{*}{$\begin{array}{l}\text { Radiometric } \\
\text { Sensitivity }\end{array}$} & PM Collection Area & COCOA (reflection area) & With 2 PMSAs \\
\hline & Vignetting & Pass and a Half & With BIA \\
\hline & $\begin{array}{l}\text { Pupil Alignment / Truant } \\
\text { Path }\end{array}$ & Fiducial lights & With 2 PMSAs \& BIA \\
\hline \multirow{3}{*}{ WFSC } & $\begin{array}{l}\text { Plate Scale } \\
\text { (tilt of single ACF \& } \\
\text { NIRCam) }\end{array}$ & Pass and a Half & With BIA \\
\hline & WFS\&C Demo & $\begin{array}{l}\text { Pass and a Half, COCOA, } \\
\text { photogrammetry }\end{array}$ & $\begin{array}{l}\text { With } 2 \text { PMSAs, SMA \& BIA, } \\
\text { limited to PMSA and SMA } \\
\text { closed loop control }\end{array}$ \\
\hline & $\begin{array}{l}\text { WFS\&C Influence } \\
\text { Functions }\end{array}$ & $\begin{array}{l}\text { Pass and a Half, COCOA, } \\
\text { photogrammetry }\end{array}$ & With 2 PMSAs, SMA \& BIA \\
\hline \multirow{3}{*}{ Functional } & $\begin{array}{l}\text { PMSA Envelope Control } \\
\text { Limit }\end{array}$ & COCOA & Yes \\
\hline & $\begin{array}{l}\text { WF Control Signal Path } \\
\text { (PMSA, SMA motion } \\
\text { control sign check test) }\end{array}$ & COCOA & With 2 PMSAs \& SMA \\
\hline & Fine Guidance Loop & Half Pass, Pass and a Half & Partial (no FGS) \\
\hline \multirow{10}{*}{ Image Quality } & PM to AOS alignment & Photogrammetry & With 1 PMSA \\
\hline & SMA to AOS alignment & Photogrammetry & Yes \\
\hline & ISIM to AOS alignment & Half Pass, Fiducial Lights & With BIA \\
\hline & PM RoC & COCOA, ADM & Partial \\
\hline & low freq PM WFE & COCOA & With 2 PMSAs \\
\hline & mid frequency PM WFE & COCOA & With 2 PMSAs \\
\hline & $\mathrm{PM}$ conic & COCOA, ADM & With 2 PMSAs \\
\hline & Ambient PMSA WFE & COCOA & With 2 PMSAs \\
\hline & $\begin{array}{l}\text { Multi-field PSF } \\
\text { (single ACF aperture, each } \\
\text { SI) }\end{array}$ & Pass and a Half & With BIA \\
\hline & SI FOV Survey & Pass and a Half & With BIA \\
\hline \multirow[b]{2}{*}{$\begin{array}{l}\text { Image Quality } \\
\text { Stability }\end{array}$} & $\begin{array}{l}\text { Thermal Distortion: PM } \\
\text { WFE \& RoC Change } \\
\text { (Figure drift of PM over } \\
\text { temp change) }\end{array}$ & COCOA & $\begin{array}{l}\text { With } 2 \text { PMSAs plus } \\
\text { additional retro-reflectors }\end{array}$ \\
\hline & $\begin{array}{l}\text { Thermal Distortion: } \\
\text { Alignment Change } \\
\text { (SMA alignment drift over } \\
\text { temp change during warm } \\
\text { up from cryo test temp) }\end{array}$ & Photogrammetry & Yes \\
\hline
\end{tabular}

Table 1: Pathfinder Testing 


\section{OGSE1}

The first of the Pathfinder tests is OGSE1 and a picture of this test configuration can be seen in Figure 4. During this test, the AOS interfaces will be cryo proof-loaded with a mass simulator. The 2 PMSA's and the SM EDU have been installed. 30 retro-reflector piston sensors will be installed on the Pathfinder backplane to mimic the missing PMSAs and will help measure the thermal distortion of the Pathfinder backplane using the COCOA. In addition, thermal sensors and cryo accelerometers will be installed. The cryo accelerometers will be used to measure the cryogenic damping of the vibrations of the backplane structure. The key pieces of optical GSE tested will be the COCOA and Photogrammetry system. In addition, this test is the first time vibration isolation of the large hanging configuration system will be checked out. Prior to running the cryo portion of the test, key GSE will first be checked out at ambient temperature to the extent practical.

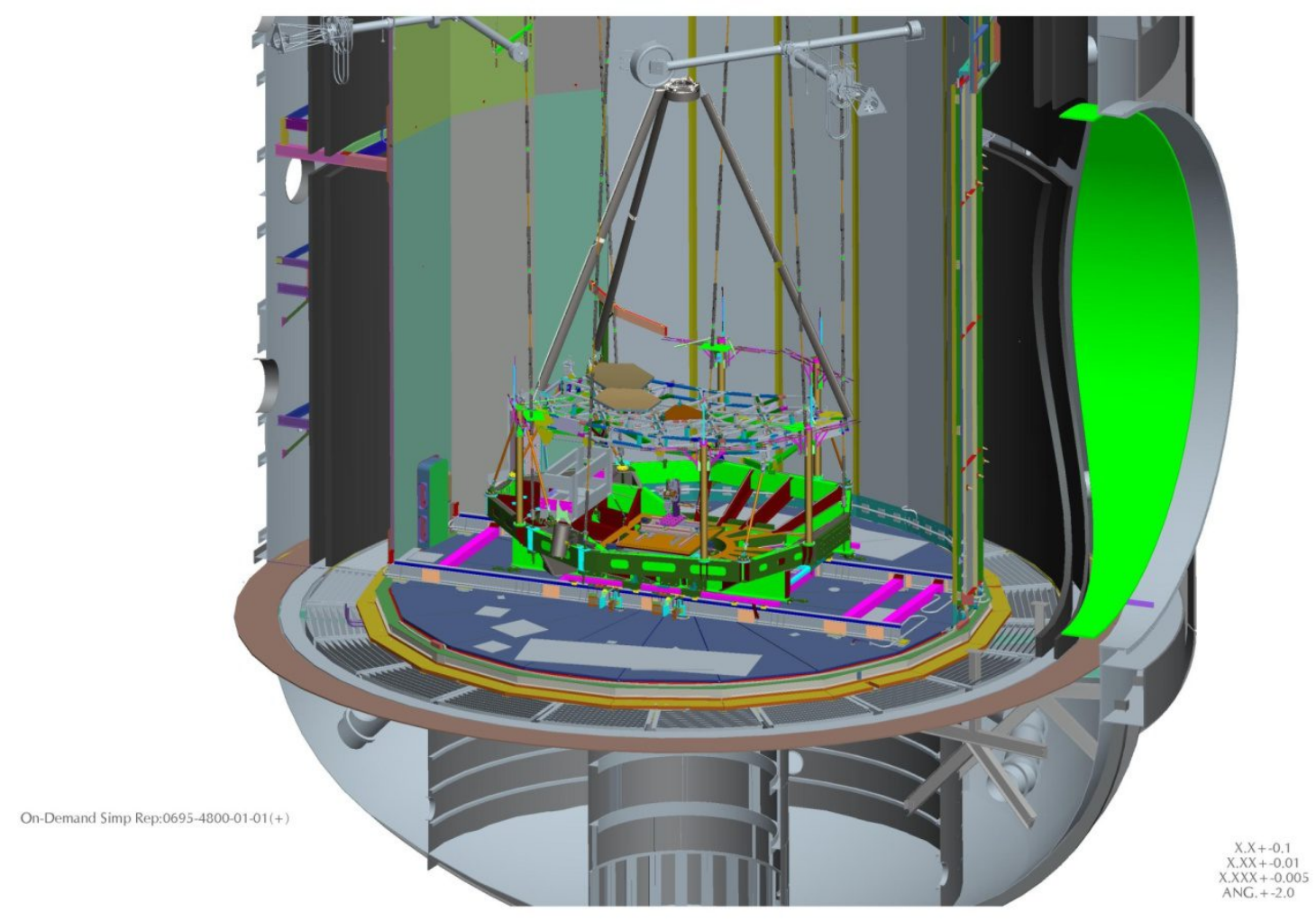

Figure 4: OGSE1

\section{OGSE2}

The second Pathfinder test is OGSE2 and a picture of this test configuration is shown in Figure 5. The key aspect of this test is that the Aft Optics System (AOS) will be installed on the Pathfinder and the AOS Source Plate Assembly (ASPA) will be installed and tested with the Beam Image Analyzer (BIA). The ASPA and BIA can be seen in Figure 6. The ASPA includes a number of fiber fed near infrared sources along with mid infrared sources that are of the same design used to test the Spitzer main camera. ASPA sources are at the Cassegrain focus of the telescope and there are both upward and downward sources. The upward sources will be pass and a half and reflect off the one ACF that will be installed for OGSE testing. The BIA has a $2 \mu \mathrm{m}$ sensitive infrared detector and translation stages along with metrology 
that will allow mapping of the aberrated images that result, and allow us to determine their locations and assess their focus and wavefront. In addition, the BIA will have a pupil imaging capability and an InSb radiometer capability sensitive to the $5 \mu \mathrm{m}$ region. The BIA will therefore act as a surrogate for the science instruments. Using the radiometer capability the BIA can be used to look for thermal straylight leaks that would be detrimental to OTIS level testing as well as checking the operation of the MIRI science instrument ASPA sources.

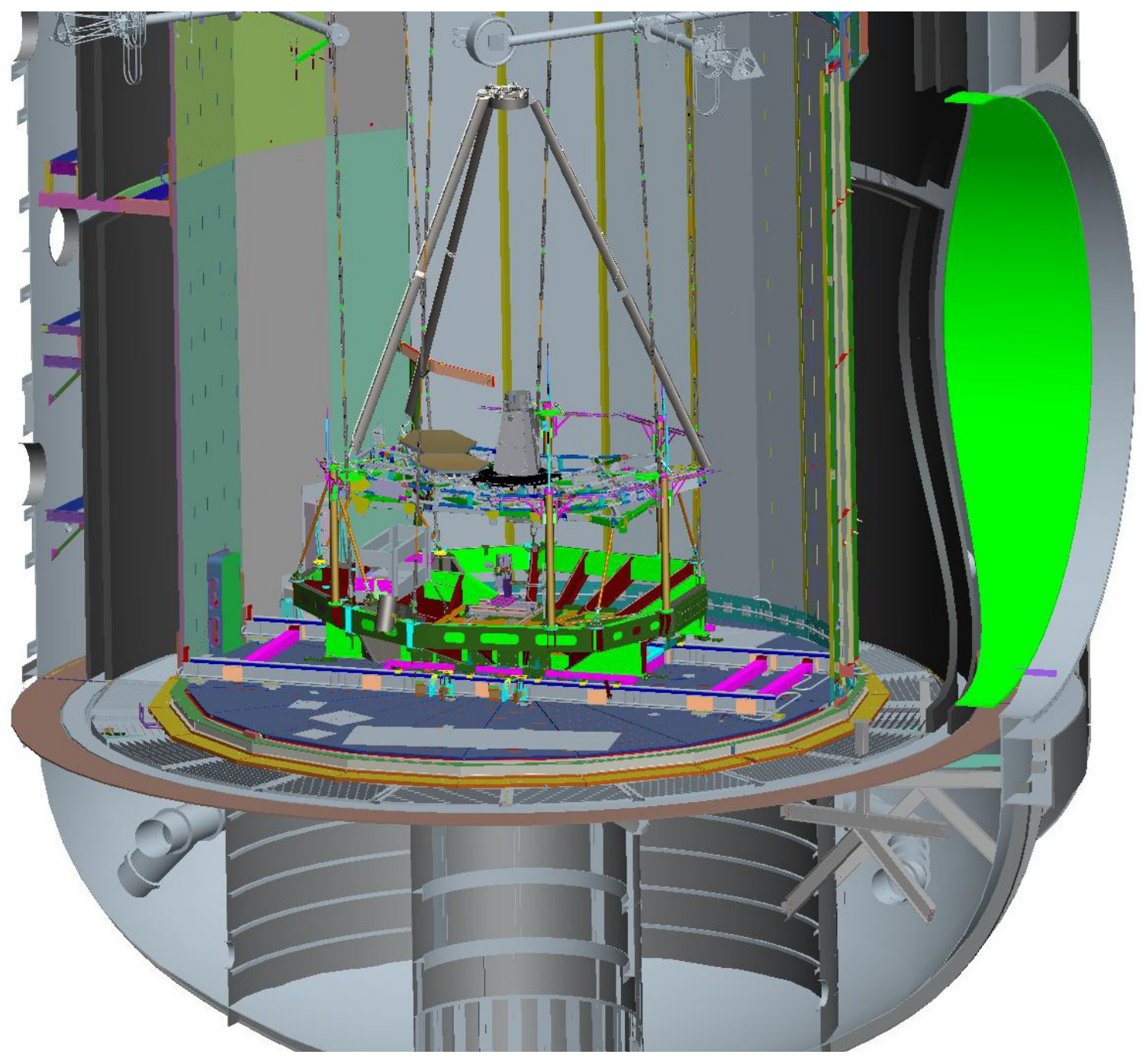

Figure 5: OGSE2 


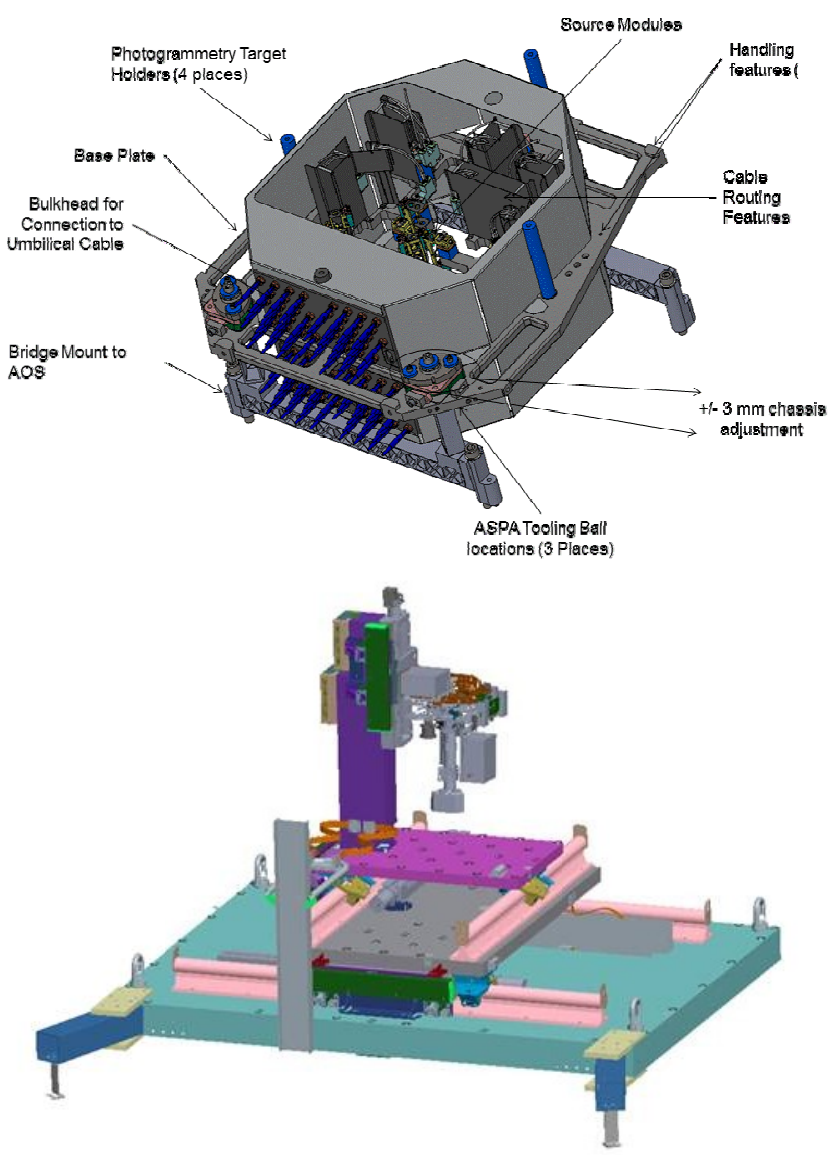

Figure 6: AOS Source Plate Assembly (ASPA) and Beam Image Analyzer (BIA)

\section{Thermal Pathfinder Test}

The third Pathfinder test is the Thermal Pathfinder Test. During this test, the flight AOS is removed but an AOS thermal simulator and mirror thermal simulators are installed along with MLI closeout. In addition, the key cryo thermal GSE for the OTIS test will be installed including the Space Vehicle Thermal Simulator (SVTS) shown in the left of Figure 7, the cryogenic cooler GSE that will be used for the MIRI in the OTIS test, and the thermal sinks for the OTIS radiators. SVTS is mechanically coupled to the HOSS which holds the telescope and includes thermal interfaces that simulate the flight interfaces. This will allow for an excellent dry run of the thermal aspects of the cooldown, warmup mirror actuator operation, and thermal balance testing. 


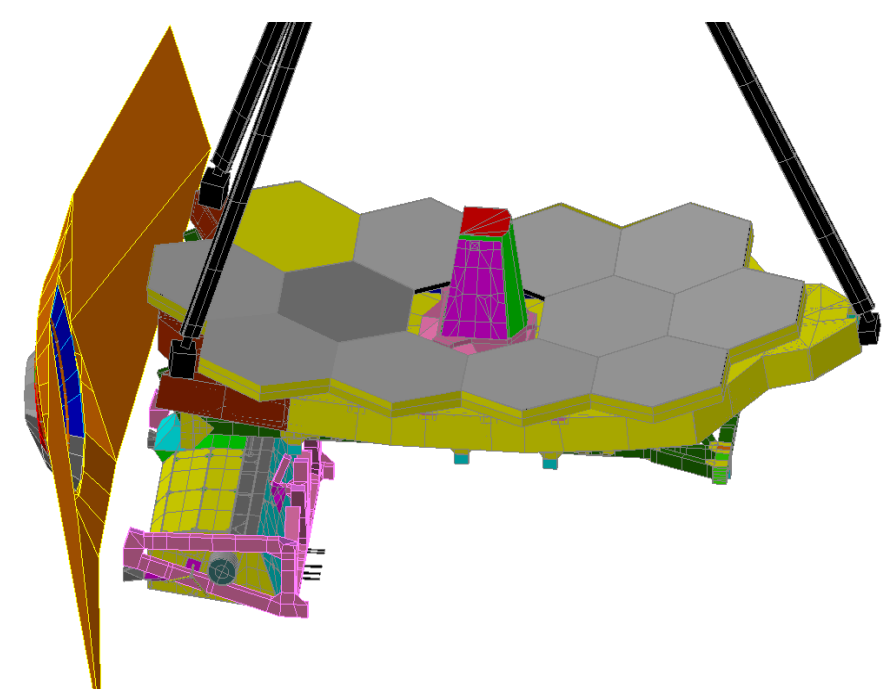

Figure 7: Thermal Pathfinder

\section{SUMMARY}

The Pathfinder structure is built and the fully assembled Pathfinder with mirrors will be complete this year. The test facility and GSE are in commissioning and will be ready for cryogenic optical testing with the Pathfinder early next year. An incremental checkout approach will be used to check out GSE and test procedures giving high confidence that the flight test will be successful. Three cryogenic tests of Pathfinder are planned to incrementally reduce the risk of the flight OTIS testing.

\section{ACKNOWLEDGEMENT}

The authors would like to thank the very large team across the country working on the JWST OTE including but not limited to key teammates NGAS, Ball Aerospace, Exelis, and Orbital/ATK, GSFC, JSC, Sigma and SAO.

\section{REFERENCES}

\footnotetext{
${ }^{i}$ Feinberg, Keski-Kuha, Atkinson, Texter, "Results, Status and Plans for the JWST OTE", SPIE Proc, (2010)

${ }^{\text {ii }}$ Feinberg et al, "New Approach to Cryogenic Optical Testing the JWST", SPIE Proc. 6265-23 (2006) ${ }^{\mathrm{ii}}$

iii Atkinson et al, "Architecting a Revised Optical Test Approach for JWST", SPIE Proc., 7010-26 (2008)

${ }^{\text {iv }}$ Kimble, et al "The integration and test program of the James Webb Space Telescope" SPIE Proc 8442-90 (2012)

"Whitman et al, “JWST's cryogenic position metrology system”, SPIE Proc 8442-91, (2012)
} 